\title{
Research Papers \\ Alkylcyanoacrylate drug carriers: I. Physicochemical characterization of nanoparticles with different alkyl chain length
}

\author{
R.H. Müller ${ }^{a}$, C. Lherm ${ }^{b}$, J. Herbort ${ }^{b}$, T. Blunk ${ }^{a}$ and P. Couvreur ${ }^{b}$ \\ ${ }^{a}$ Department of Pharmaceutics and Biopharmaceutics, University of Kiel, D-2300 Kiel (Germany) and ${ }^{b}$ Université de Paris-Sud, \\ Laboratoire de Pharmacie Galénique et Biopharmacie, UA CNRS 1218, Faculté de Pharmacie, Chatenay-Malabry (France)
}

(Received 2 August 1991)

(Modified version received 25 November 1991)

(Accepted 26 November 1991)

Key words: Cyanoacrylate nanoparticle; Surface charge; Zeta potential; Surface hydrophobicity; Polymer degradation; Nanoparticle coating

\section{Summary}

Alkylcyanoacrylate particles were physico-chemically characterized in terms of size, surface charge, zeta potential, interaction with charged serum components and surface hydrophobicity as relevant parameters influencing the in vitro interaction with cells in culture and the in vivo organ distribution and fate after intravenous administration. Methyl-, ethyl-, isobutyl- and isohexylcyanoacrylate particles were found to be very similar with regard to these properties. Large differences existed with regard to their degradation behaviour. The type of degradation (surface erosion) was determined by photon correlation spectroscopy, and the degradation velocity was evaluated using a turbidimetric assay. Surface modification of the particles by a polymer coating affected neither the type nor the velocity of the degradation.

\section{Introduction}

Polyalkylcyanoacrylate (PACA) particles are of interest as possible carriers for controlled drug delivery (Couvreur, 1988). A doxorubicin-containing formulation for human use has been developed (Verdun et al., 1986), which is similar to other particulate carrier systems presently undergoing clinical trials (Delgado et al., 1989). The introduction of PACA particles into therapy is

Correspondence: R.H. Müller, Dept of Pharmaceutics and Biopharmaceutics, University of Kiel, D-2300 Kiel, Germany. hindered by their possibly toxic degradation products of alcohol, acrylic acid and formaldehyde (Vezin and Florence, 1980; Lenaertz et al., 1984). The unresolved question of toxicity limits their use at present in humans to severe diseases such as cancer. The benefits of controlled delivery of drugs such as doxorubicin outweigh possible side effects.

The major way for particle administration is the intravenous route. After i.v. injection the particles will be recognized as being foreign and be taken up mainly by the macrophages of the liver (60\%) and spleen (Grislain et al., 1983). The recognition process and the subsequent clearance 
by macrophage populations depend on the physico-chemical surface properties of the particles. Factors promoting rapid clearance from the blood by reticuloendothelial macrophages are a high particle charge (Wilkins and Myers, 1966), a hydrophobic surface (Van Oss et al., 1975) or a surface which activates complement (Kazatchkine and Carreno, 1988). A hydrophilic surface reduces opsonization in the blood but only if no complement-activating groups are present (Müller, 1991). Macrophage uptake can be eliminated by controlled modification of the particle surface (Müller, 1989; Müller and Heinemann, 1989). Coating of particles with ethoxylated polymers by an adsorption process can eliminate the macrophage uptake leading to particles which circulate in the blood (Illum et al., 1987). Such particles are possible carriers for the controlled release of drugs (e.g., peptides). Controlled surface modification can also be employed to localize particles in other tissues, e.g., bone marrow (Illum and Davis, 1987), or to shift the ratio of liver and spleen uptake (Müller, 1991). Taken as a whole, the above results showed clearly that the amount of particles localized in a tissue is determined by their surface properties. Modification of the surface reduces the extent of capture by the macrophage system and therefore exerts an influence on cytotoxicity. In addition, the modification of biodegradable particles could alter the velocity of biodegradation and subsequent release of degradation products. Therefore, the aim of this paper was to characterize the particles with regard to both surface properties and polymer degradation in order to gain a better comprehension of the interactions between cells and nanoparticles.

\section{Materials and Methods}

\section{Materials}

Methyl- and ethylcyanoacrylate were provided by Loctite Ltd (Ireland), isobutylcyanoacrylate was purchased from Sigma (U.S.A.) and isohexylcyanoacrylate was obtained from Sopar (Belgium). The abbreviations used in the subsequent text are as follows: (monomers) MCA, methylcyanoacrylate; ECA, ethylcyanoacrylate; IBCA, isobutyl- cyanoacrylate; IHCA, isohexylcyanoacrylate; (polymers) PMCA, polymethylcyanoacrylate; PECA, polyethylcyanoacrylate; PIBCA, polyisobutylcyanoacrylate; PIHCA, polyisohexylcyanoacrylate.

Propidium iodide (PI) as fluorescent marker was purchased from Sigma (U.S.A.). Poloxamine 908 and Poloxamer 188 and 407 were obtained from BASF (Wyandotte, U.S.A.).

Cell culture medium (MEM) and related chemicals were purchased from Flobio (Paris, France), and fetal calf serum from IBF (Paris, France).

\section{Methods}

Polyalkylcyanoacrylate particles were polymerised as described before (Lherm et al., 1989). For preparation of polyethylcyanoacrylate particles with incorporated propidium iodide (PECA$\mathrm{PI})$, the marker was added during the polymerization of the monomer (Müller et al., 1991).

The particle surface was modified by the adsorption of Poloxamine 908 and Poloxamer 407. The nanoparticle suspension $(1 \% \mathrm{w} / \mathrm{w})$ was mixed with an equal volume of a polymer solution ( $1 \%$ $\mathrm{w} / \mathrm{w}$ ) and incubated overnight (final concentration of polymer after mixing: $0.5 \%$ ). The polymer concentration of $0.5 \%$ was chosen in order to be well above the concentration at the plateau of the adsorption isotherms of about $0.005-0.01 \%$ (Kayes and Rawlins, 1979). For the degradation experiments the particles were diluted with the degradation medium. Such dilution did not cause desorption of the adsorbed polymer layers. The thickness of the coating layers remained unchanged as determined by PCS. The stability of the coating layers in water and in serum has been described in detail elsewhere (Wallis and Müller, 1990).

Particle size and the width of the size distribution were determined by photon correlation spectroscopy (PCS) (Cummins and Pike, 1973, 1976) using a Malvern Spectrometer in connection with a Malvern 4-bit Correlator (Malvern Instruments, Malvern, U.K.). The width of the size distribution is characterized by a polydispersity index (Koppel, 1972) ranging from 0 to 0.5 . An index of 0 corresponds theoretically to a monodisperse par- 
ticle population. For polystyrene latex particles which are regarded as being monodisperse, values between 0.03 and 0.06 were obtained. An index of 0.1 corresponds to a narrow distribution, and no defined logarithmic normal distribution can be attributed to an index above 0.5 (very broad distribution).

The surface charge was determined by conductimetry (basic titration with $\mathrm{NaOH}, 25^{\circ} \mathrm{C}$ ) and expressed in $\mu$ eq. $/ 1$ nanoparticle suspension (1\% $\mathrm{w} / \mathrm{w}$ ) or $\mu \mathrm{C} / \mathrm{cm}^{2}$ (Lherm, 1990).

The zeta potential was determined by performing electrophoresis measurements using a Malvern ZetaSizer II (Malvern Instruments, Malvern, U.K.). The particle velocity in the electrical field was evaluated through laser Doppler anemometry (Cummins and Pike, 1976). Measurements were made in different media (distilled water, $0.01 \mathrm{M}$ citrate-phosphate buffer, physiological salt solution $(0.9 \% \mathrm{NaCl}$ solution) and in serum). The potential was calculated on the basis of the Helmholtz-Smoluchowski equation (Hunter, 1981).

The surface hydrophobicity was characterized by measuring the adsorption layer of Poloxamer 407 and Poloxamine 908 on the particles. The thickness was shown to increase with increasing surface hydrophobicity of the particles (Müller, 1991). The coating layer thickness was determined by PCS.

To determine the type of degradation, degradation experiments were performed in distilled water containing $10^{-4}$ to $1 \mathrm{~N} \mathrm{NaOH}$. PCS was carried out for monitoring of the alterations in size and polydispersity of the particles with time.

To measure the degradation velocity, turbidimetry (absorption at $400 \mathrm{~nm}$, Uvikon 810 spectrometer, Kontron, Germany) was employed. Particles were subjected to degradation in cell culture medium containing 5\% fetal calf serum (FCS). Addition of particles to the cell culture medium causes particle aggregation due to the reduction in zeta potential at higher electrolyte concentrations. To eliminate aggregation, particles were sterically stabilized by coating with Poloxamine 908 or Poloxamer 407. The half-life of degradation, $t_{50 \%}$, was defined as the time required to reduce the extent of the initial absorption to $50 \%$ (Müller et al., 1990).

\section{Results and Discussion}

\section{Characterization of particle size and distribution}

The size of nanoparticles varied from 132 to $314 \mathrm{~nm}$ whereby no linear relationship between size and alkyl chain length existed (Table 1). The size was mainly determined by the polymerization conditions, e.g., the type and concentration of surfactant employed. Alterations in size were also achieved when monomers obtained from different suppliers were used for particle polymerization. Two types of isobutylcyanoacrylate particles having different sizes were prepared by varying the surfactant concentration (Poloxamer 188, Table 1) in order to examine the possible influ-

TABLE 1

Particle size and polydispersity index of alkylcyanoacrylate particles produced by variation of surfactant / stabilizer type and surfactant concentration

\begin{tabular}{|c|c|c|c|c|}
\hline \multirow{2}{*}{$\begin{array}{l}\text { Alkyl } \\
\text { chain }\end{array}$} & \multirow{2}{*}{$\begin{array}{l}\text { Size } \\
(\mathrm{nm})\end{array}$} & \multirow{2}{*}{$\begin{array}{l}\text { Polydis- } \\
\text { persity } \\
\text { index }\end{array}$} & \multicolumn{2}{|c|}{ Surfactant/stabilizer } \\
\hline & & & Type & $\begin{array}{l}\text { Concen- } \\
\text { tration }(\%)\end{array}$ \\
\hline Methyl & 204 & 0.030 & Tween 20 & 0.36 \\
\hline Ethyl & 314 & 0.026 & dextran 70 & 1.0 \\
\hline Isobutyl & 132 & 0.120 & dextran 70 & 1.0 \\
\hline Isohexyl & 136 & 0.013 & dextran 70 & 1.0 \\
\hline Isobutyl & 50 & 0.103 & Poloxamer 188 & 2.0 \\
\hline Isobutyl & 200 & 0.121 & Poloxamer 188 & 0.1 \\
\hline
\end{tabular}


ence of size on the cytotoxicity of the particles as determined in cell cultures (Lherm et al., 1992). The size also influences the total number of particles and therefore the total mass of polymer taken up by the cells (Johnson et al., 1986).

The polydispersity index (PD) indicated a very narrow size distribution for methyl-, ethyl- and isohexylcyanoacrylate particles, similar to that of monodisperse standard latex (PD approx. 0.05), with that of isobutylcyanoacrylate particles being slightly broader (PD about 0.1 ). The difference in width of the distribution can be ascribed to the conditions employed during the process of polymerization $(0.1 \%$ dextran 70$)$, which possibly may have been less than optimal for the polymerization of IBCA. However, the experimental conditions used were maintained constant for each of the three polymers (PECA, PIBCA and PIHCA) in order to restrict possible differences in the surface properties between the particles to a minimum. Nevertheless, in the case of the preparation of methylcyanoacrylate particles, it was necessary to replace dextran 70 by Tween 20 to achieve an acceptable size distribution.

\section{Particle surface charge}

Titration of the particle suspensions using $\mathrm{NaOH}$ yielded a charge of $-43,-17$ and -17 $\mu$ eq. $/ 1$ for PECA, PIBCA and PIHCA nanoparticle suspensions, respectively. This corresponded to a surface charge of $-2.12,-0.37$ and -0.42 $\mu \mathrm{C} / \mathrm{cm}^{2}$ calculated on the basis of the PCS diameter of the investigated particles. The measured surface charge was relatively low compared with

\section{TABLE 2}

$\zeta$-potentials of alkyl-cyanoacrylate particles in distilled water, $0.01 \mathrm{M}$ citrate-phosphate buffer at $\mathrm{pH} 7.4$ and physiological $\mathrm{NaCl}$ solution $(0.9 \%)$

\begin{tabular}{llll}
\hline \multirow{2}{*}{$\begin{array}{l}\text { Alkyl } \\
\text { chain }\end{array}$} & \multicolumn{3}{l}{$\zeta$-potential $(\mathrm{mV})$} \\
\cline { 2 - 4 } & $\begin{array}{l}\text { Distilled } \\
\text { water }\end{array}$ & $\begin{array}{l}0.01 \mathrm{M} \\
\text { buffer }\end{array}$ & $\begin{array}{l}0.9 \% \mathrm{NaCl} \\
\text { solution }\end{array}$ \\
\hline Methyl & -26.0 & -10.5 & -0.4 \\
Ethyl & -20.5 & -0.8 & +0.6 \\
Isobutyl & -6.3 & -0.9 & +0.9 \\
Isohexyl & -11.2 & -6.3 & +0.5 \\
\hline
\end{tabular}

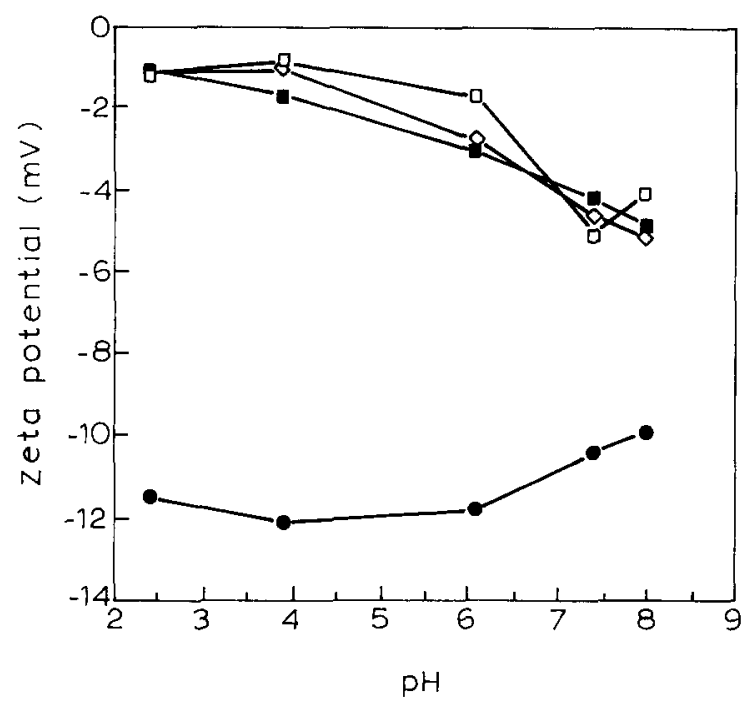

Fig. 1. $\zeta$-potential vs pH profiles of PMCA ( $\bullet$, PECA $(\boldsymbol{\square})$, PIBCA $(\diamond)$ and PIHCA $(\square)$ particles in $0.01 \mathrm{M}$ citrate-phosphate buffer.

particles such as carboxylated and hydroxylated polystyrene latex particles. Indeed, the surface charges were in the range of above $0.1-0.2$ meq. $/ g$ polymer $(200 \mathrm{~nm}$ particles) (Polysciences product catalogue). This corresponds to a charge of $1000-2000 \mu \mathrm{eq} . / \mathrm{l}$ of a $1 \%(\mathrm{w} / \mathrm{w})$ particle suspension and a surface charge between 33 and $66 \mu \mathrm{C} / \mathrm{cm}^{2}$.

\section{Zeta potential of particles}

Measurements of the $\zeta$-potential were performed as an alternative means of evaluating particle surface charge. The $\zeta$-potential (Hunter, 1981) is correlated with the Nernst potential of the surface, which means that, in most cases, the $\zeta$-potential is proportional to the Nernst potential.

Electrophoresis measurements in distilled water revealed the highest potentials for PMCA and PECA, while PIBCA and PIHCA showed distinctly lower values (Table 2). This is in agreement with the measured surface charge.

Increasing the electrolyte concentration compressed the diffuse layer (Hunter, 1981) and therefore reduced the $\zeta$-potential in $0.01 \mathrm{M}$ citrate-phosphate buffer (Table 2 ). The $\zeta$-potential vs $\mathrm{pH}$ profiles demonstrated a minimum $\zeta$-potential at low $\mathrm{pH}$ due to the lower degree of dissoci- 
TABLE 3

$\zeta$-potentials of alkylcyanoacrylate particles in culture medium with $5 \%$ fetal calf serum, in serum and of senum-coated alkylcyanoacrylate particles in distilled water (values for latex particles taken from Müller, 1991)

\begin{tabular}{llll}
\hline $\begin{array}{l}\text { Alkyl } \\
\text { chain }\end{array}$ & \multicolumn{2}{l}{$\zeta$-potential $(\mathrm{mV})$} & \\
\cline { 2 - 4 } & $\begin{array}{l}\text { In culture } \\
\text { medium with } \\
10 \% \text { serum }\end{array}$ & In serum & $\begin{array}{l}\text { Of serum-coated } \\
\text { particles } \\
\text { in distilled } \\
\text { water }\end{array}$ \\
\hline Methyl & -1.0 & -2.7 & -4.9 \\
Ethyl & -1.9 & -2.5 & -5.4 \\
Isobutyl & -0.3 & -1.8 & -3.6 \\
Isohexyl & +0.5 & -3.7 & -5.0 \\
60 nm & & & \\
$\quad$ latex & - & -15.3 & -25.7 \\
Latex + & & & \\
Poloxamine & & -5.2 & -3.9 \\
908 coat & - & & \\
\hline
\end{tabular}

ation of free acrylic acid groups at this $\mathrm{pH}$ (Fig. 1). Further increase in the electrolyte concentration on using $\mathrm{NaCl}$ solution at physiological concentration led to zero $\zeta$-potentials for all particles (Table 2). As a consequence, no differences in the interaction with cells (culture medium with physiological salt concentration) as a result of charge are expected for the different cyanoacrylate particles. In contrast, the particle-cell interaction could be partly determined by particle charges acquired through the adsorption of charged serum components (Müller, 1991) present in the culture medium.

\section{Interaction with serum components}

That physiological salt concentrations reduced the $\zeta$-potential of the particles to zero (Table 2) was confirmed by carrying out measurements in culture medium containing 5\% serum (Table 3). Determinations of $\zeta$-potential in serum yielded potentials ranging from -2 to $-4 \mathrm{mV}$, indicating a low degree of uptake of charged serum components. In serum, $\zeta$-potentials were found to be very similar to those of Poloxamine 908-coated $60-\mathrm{nm}$ polystyrene latex particles (Table 3) (Müller, 1991). The Poloxamine 908 coat creates a hydrophilic surface with a 'dysopsonic effect', which signifies that the uptake of phagocytosisenhancing serum opsonins is low in extent. Strong adsorption of charged serum components led to particle charges in the range of $-15 \mathrm{mV}$, e.g., for polystyrene latex particles undergoing rapid phagocytosis by macrophages in vivo (Table 3 ).

To differentiate between particles having similar potentials in serum, the electrophoresis measurements of serum-coated particles were performed in distilled water. Due to the reduction in electrolyte concentration, decompression of the diffuse layer occurred, resulting in an increase in the $\zeta$-potential. Such an increase magnifies possible differences between similarly charged particles in serum (Müller, 1991). In distilled water, the $\zeta$-potential of the uncoated latex particles increased whereas only a slight change was found to occur for the coated latex and cyanoacrylate particles. From these results, the four tested alkylcyanoacrylate particle types appeared to be similar in terms of adsorption of charged serum components. Considerable differences in the number of particles internalized by cells due to variations in the extent of opsonization were therefore not expected to occur.

\section{Surface hydrophobicity}

Surface hydrophobicity can be determined by measuring the adsorption of hydrophobic compounds and calculating an affinity constant or measuring contact angles. Alternatively, a convenient method involves the determination of the thickness of adsorbed polymer layers by PCS and using this parameter as a measure of surface hydrophobicity (Müller, 1991). Therefore, the layer thickness of block polymers (Poloxamine 908 and Poloxamer 407) adsorbed on cyanoacrylic nanoparticles was investigated.

Incubation of the cyanoacrylate particles with 1.0\% Poloxamer 407 solution led to the adsorption of polymer on all particles as demonstrated by the removal of aggregates (reduction in polydispersity index). For the hydrophobic methyl-, ethyl- and isobutylcyanoacrylate particles, the coating layer was too thin to be measured by PCS. However, a layer of $64 \AA$ was observed for isohexylcyanoacrylate particles, indicating that 
these were the most hydrophobic of those examined.

Subsequent to incubation with Poloxamine 908, coating layers were measurable for all four types of particles. The layers were found to be of similar thickness in the case of PMCA, PECA and PIBCA $(47,52$ and $40 \AA$, respectively), indicating the degree of hydrophobicity to be compa-
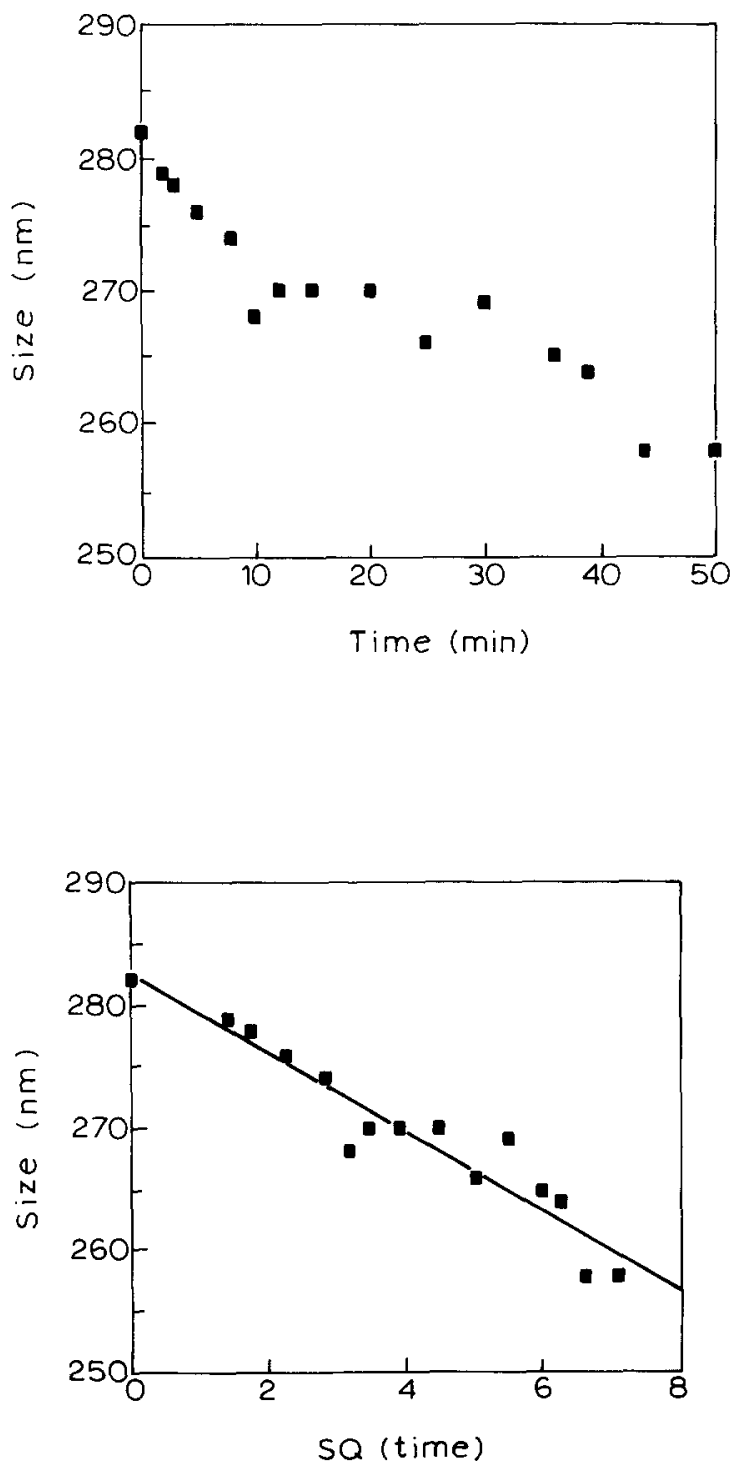

Fig. 2. Degradation of PECA nanoparticles in $1 \times 10^{-4} \mathrm{M}$ $\mathrm{NaOH}$ plotted vs time on a linear time scale (upper panel) and vs the square root of time (lower panel). The size decreased without a lag time ( $24 \mathrm{~nm}$ decrease within $50 \mathrm{~min}$ ).

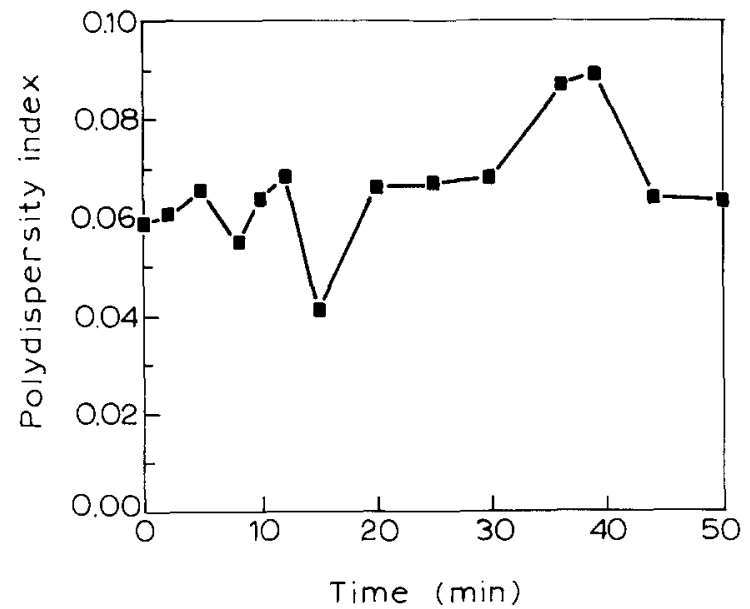

Fig. 3. Polydispersity index measured for the degradation of PECA in $1 \times 10^{-4} \mathrm{M} \mathrm{NaOH}$ during the initial $50 \mathrm{~min}$ (cf. Fig. 2).

rable in magnitude among these three particle types. A modest increase in thickness was observed for PIHCA ( $75 \AA$ ), due to the surface of this particle type being slightly more hydrophobic in nature. This is correlated with the degree of surface hydrophobicity of alkylcyanoacrylate polymer films as characterized on the basis of determinations of contact angles. The values obtained for these angles were rather close, however, a slight increase with greater alkyl chain length became evident (Kreuter, 1983). For the purpose of comparison, a layer thickness of $120 \AA$ has been obtained previously, using hydrophobic polystyrene latex particles (Müller, 1991).

Determination of the type of nanoparticle degradation

To determine the type of degradation (bulk or surface degradation), the process was followed by performing PCS measurements. In the case of surface degradation, the particle size should show an immediate increase whereby the polydispersity index remains constant. If bulk degradation dominates, a lag period should occur, preceding the decrease in mean size due to disintegration of the particles. This process of disintegration would lead to a more heterogeneous distribution of particle sizes and consequently to an increase in the polydispersity index. 
Incubation of PECA in $10^{-4} \mathrm{~N} \mathrm{NaOH}$ led to an immediate, continuous decrease in size of the particles without a delay time (Fig. 2). Within 50 min, a reduction in size by $24 \mathrm{~nm}$ was attained whereas the polydispersity index remained unchanged at a mean value of $0.064( \pm 0.013)$. The fluctuations were within the range observed typically for the PCS polydispersity index (Fig. 3). This clearly indicated the process to be predominantly that of surface degradation.

Incorporation of propidium iodide had no effect on the degradation velocity of PECA particles (Fig. 4). As a consequence of the conditions employed during preparation, the propidium iodide PECA particles (PECA-PI) were slightly larger than those of PECA. However, the size decrease due to surface erosion was found to be identical in extent for both types of particles ( 24 $\mathrm{nm}$ within $50 \mathrm{~min}$ ). Therefore, the incorporation of fluorescent markers at a low payload does not interfere with the measurement of phenomena in relation to the degradation velocity of particles.

Coating of the PECA-PI particles with Poloxamine also had no influence on the velocity of degradation (Fig. 4). The coating of PECA nanoparticles with surface-active block polymers for targeting purposes increases the hydrophilicity and wettability of the surface (Müller, 1991).

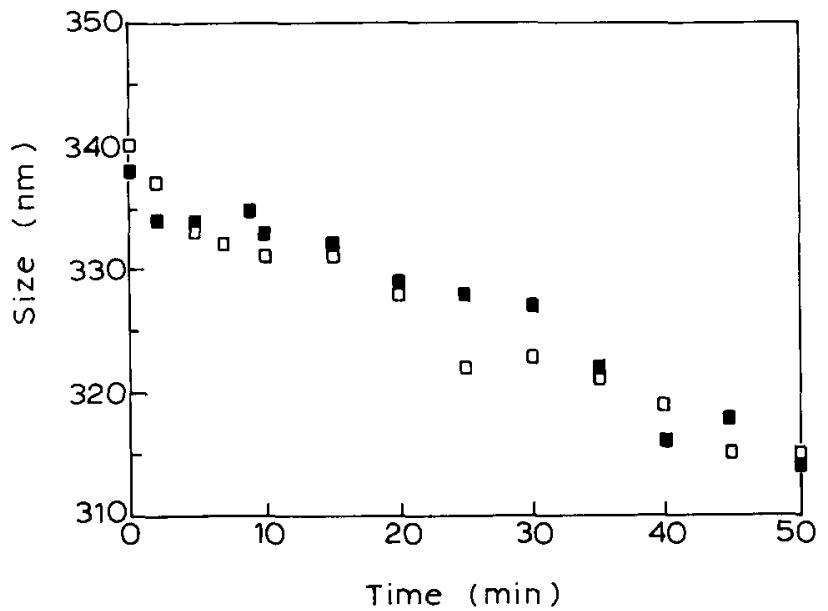

Fig. 4. Degradation of PECA-PI particles ( $\boldsymbol{u})$ and Poloxamine 908-coated PECA-PI particles $(\square)$ in $1 \times 10^{-4} \mathrm{M} \mathrm{NaOH}$. The size decreased by a total of 24 and $25 \mathrm{~nm}$, respectively, within $50 \mathrm{~min}$.

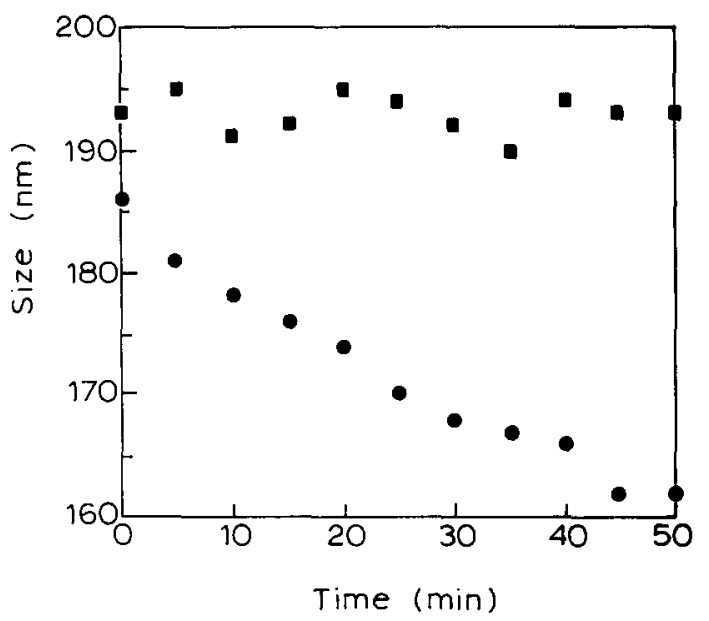

Fig. 5. Degradation of PIHCA nanoparticles in $1 \times 10^{-4} \mathrm{M}$ (ש) and $1 \times 10^{-2} \mathrm{M}(\bullet) \mathrm{NaOH}$. No measurable degradation was observed in $1 \times 10^{-4} \mathrm{M} \mathrm{NaOH}$. A 100 -fold greater concentration of $\mathrm{NaOH}$ was needed to achieve a velocity of degradation identical $(24 \mathrm{~nm} / 50 \mathrm{~min})$ to that of PECA particles.

However, this does not result in the acceleration of carrier degradation.

Incubation of PIHCA particles at $10^{-4} \mathrm{NaOH}$ led to no detectable size decrease within the first $50 \mathrm{~min}$ (Fig. 5). In fact, the size and polydispersity index remained unchanged over an observation period of 24 h. A 100 -fold increase in $\mathrm{NaOH}$ concentration was necessary in order to achieve an identical degradation velocity to that of PECA (24 nm during the first $50 \mathrm{~min}$ ) (Fig. 5). The PIHCA particles also degraded via a process of surface erosion but at a much slower rate compared to PECA.

Monitoring the size of PIHCA particles in 0.01 $\mathrm{N} \mathrm{NaOH}$ over a period of $4 \mathrm{~h}$ surprisingly revealed a plateau effect at $125 \mathrm{~nm}$ followed by a slight increase to $135 \mathrm{~nm}$ close to $4 \mathrm{~h}$ (Fig. 6). Before attaining a plateau, the PCS count rate displayed a considerable decrease, indicating that almost $100 \%$ of the particles had undergone degradation. The solution appeared clear without any Tyndall effect; in the UV spectrometer, zero absorption was measured. Although the process of degradation had reached completion, a size reading was still evident due to the sensitivity of the PCS in detecting a few remaining particles. 
The nanoparticle population seemed to contain a few extremely slowly degrading particles. These are possibly relatively large particles or aggregates formed by flocculation of small particles. That flocculation did occur was demonstrated by the increase in size (Fig. 6, upper) and in polydispersity index towards $4 \mathrm{~h}$ (Fig. 6, lower). The presence of dust might also contribute to such an increase, since PCS gives a mean diameter for all particulates in the sample (i.e., nanoparticles and particulate contaminations). With decreasing number of nanoparticles, the measured mean size will be more strongly determined by the contribution of the remaining larger particles and the
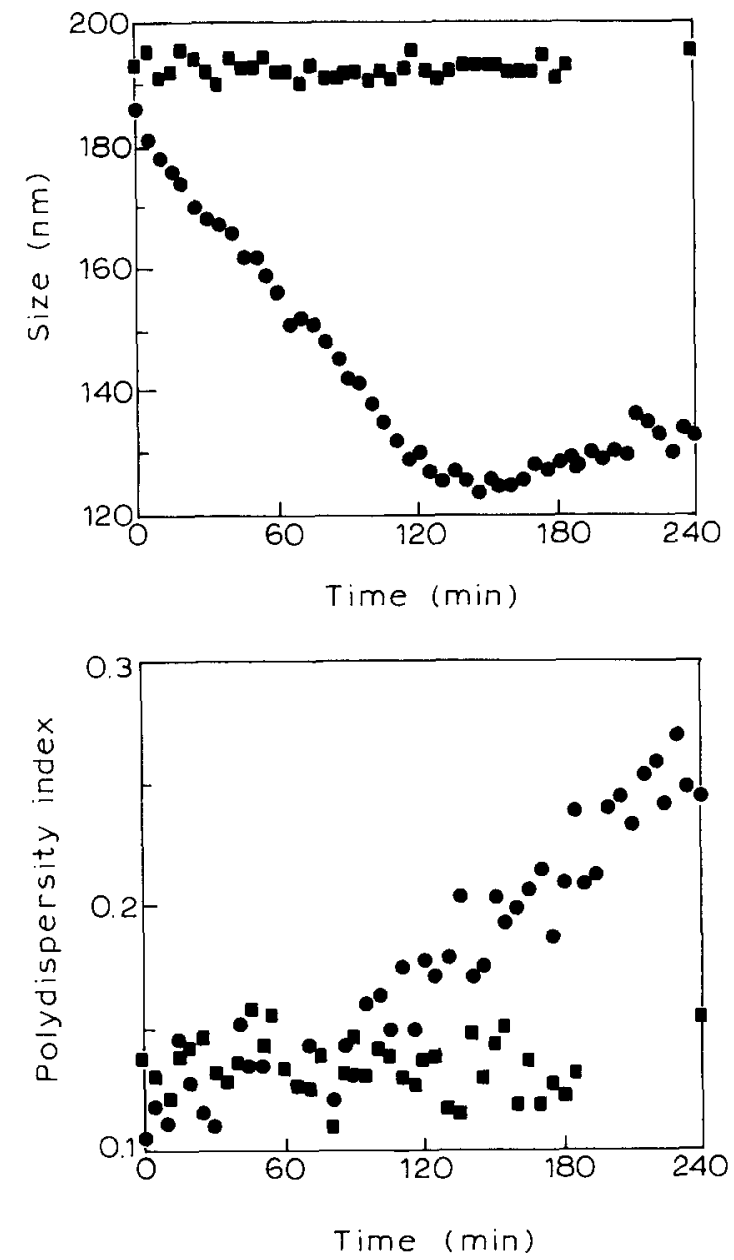

Fig. 6. PCS size (upper panel) and polydispersity index (lower panel) of PIHCA nanoparticles dispersed in $1 \times 10^{-2} \mathrm{M}(\bullet)$ and $1 \times 10^{-4} \mathrm{M}(\mathbf{\square}) \mathrm{NaOH}$ over a period of $240 \mathrm{~min}$.
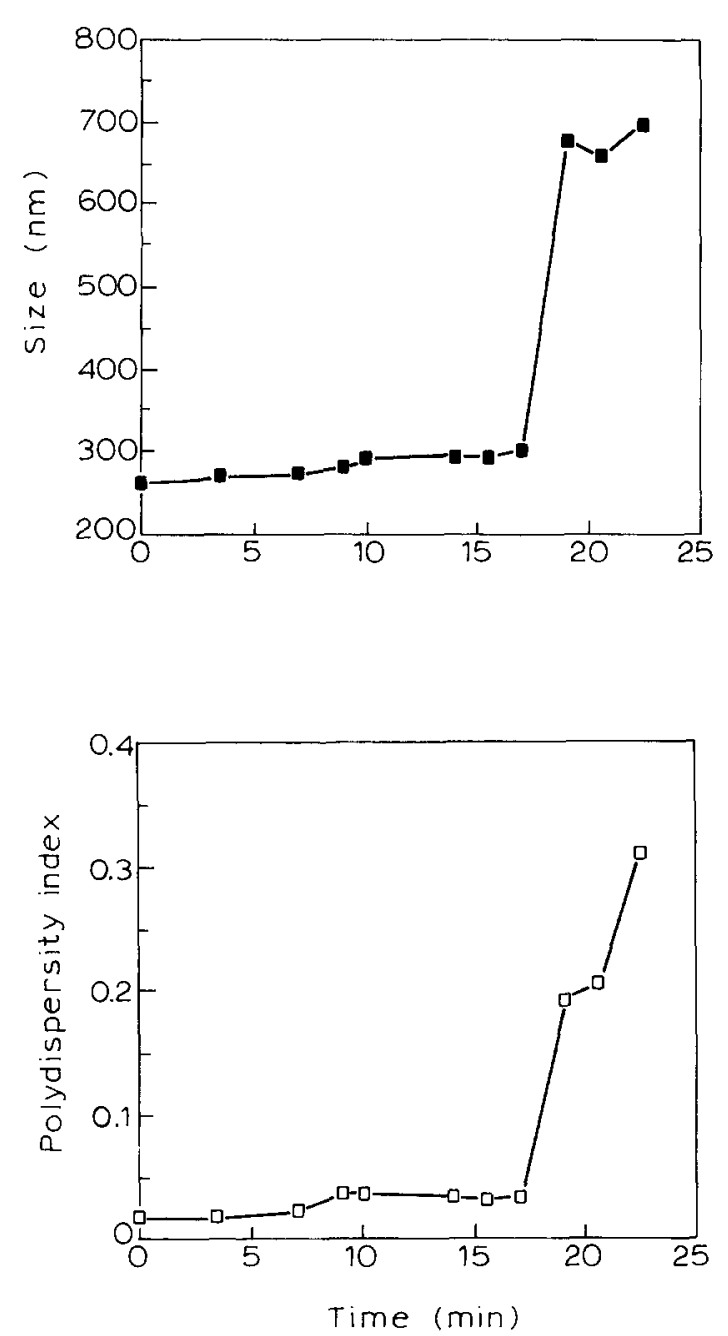

Fig. 7. PCS size (upper panel) and polydispersity index (lower panel) of PIBCA nanoparticles in $1 \mathrm{M} \mathrm{NaOH}$. The high concentration of electrolyte results in flocculation, as indicated by the rapid increase in size and polydispersity index.

particulate contaminants.

At low electrolyte concentration, distortions due to flocculation do not occur. The size and polydispersity of PIHCA particles remained unchanged during incubation in $10^{-4} \mathrm{~N} \mathrm{NaOH}$ (Fig. 6). At higher ionic strength, the size increase as a result of flocculation was thus larger than the size decrease due to degradation. Therefore, no initial decay took place but an immediate increase in size was observed for PIBCA particles in $1 \mathrm{~N}$ $\mathrm{NaOH}$ (Fig. 7). The size increase was due to 
particle flocculation, as shown by the correspondingly larger polydispersity index (Fig. 7, lower). It can also be seen that the flocculation-induced distortions were less important during the initial phase.

From these results, it was concluded that size measurements by PCS were suitable in order to determine the type of degradation at low electrolyte concentrations and during the initial phase (when distortions due to flocculation are negligible). To determine the time of completion for degradation (especially at physiological salt concentrations), the PCS count rate should be taken which corresponds to a turbidimetric measurement rather than the calculated size value. However, the count rate was found to be more sensitive towards particulate contamination than the spectrometer.

\section{In vitro degradation of particles}

To avoid problems in determining the time required for degradation to reach completion using PCS, turbidimetric measurements were employed (Müller et al., 1990). The fastest degradation was observed for methyl- and ethylcyanoacrylate particles (Fig. 8).

PIBCA and PIHCA particles degraded much more slowly over a few hours (Fig. 9). The slow decrease in absorption was thought to be due not

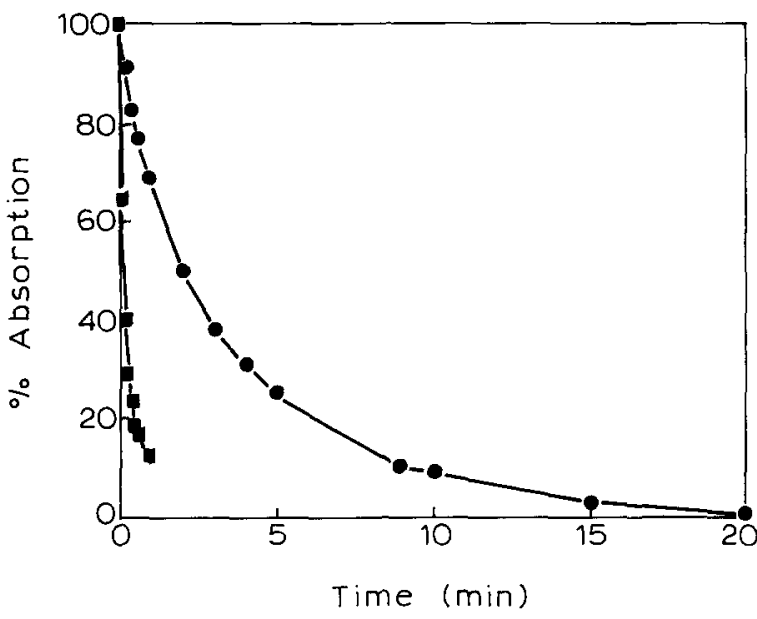

Fig. 8. Degradation of PMCA ( $\boldsymbol{\square})$ and PECA (•) nanoparticles in cell culture medium containing $5 \%$ fetal calf serum. The course of degradation was monitored by measuring the absorption at $400 \mathrm{~nm}$.

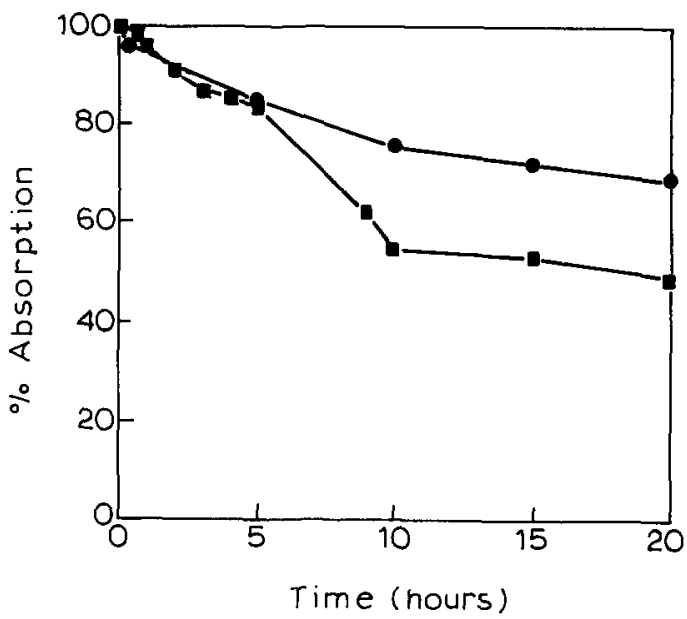

Fig. 9. Degradation of uncoated PIBCA ( $\boldsymbol{0}$ ) and PIHCA (•) nanoparticles in cell culture medium containing $5 \%$ fetal calf serum.

only to slow degradation but also to a simultaneous increase in the extent of absorption due to particle aggregation. The high concentration of electrolyte in the culture medium led to the formation of larger particle aggregates accompanied by an increase in absorption. The $t_{50 \%}$ values of more than $10 \mathrm{~h}$ determined by extrapolation of the curve therefore appeared to be too long (Table 4). Particle aggregation was thus reduced by steric stabilization of the particles using Poloxamer 407 or Poloxamer 908. As judged on the basis of the PCS data, coating with these polymers did not appear to interfere with the degradation process (no acceleration of degradation due to increased wettability of the particles). After the elimination of distortions by coating, the rate of absorption decreased much more rapidly

\section{TABLE 4}

Half-life for cyanoacrylate particle degradation in cell culture medium at $p H 7.4$ (particles were sterically stabilized (final column) in order to eliminate distortions due to particle aggregation)

\begin{tabular}{lcc}
\hline Particles & \multicolumn{2}{c}{ Half-life of degradation for particles } \\
\cline { 2 - 3 } & Non-stabilized & Stabilized \\
\hline PMCA & $<0.5 \mathrm{~min}$ & $<0.5 \mathrm{~min}$ \\
PECA & $2 \mathrm{~min}$ & $2 \mathrm{~min}$ \\
PIBCA & $12 \mathrm{~h}$ & $4.6 \mathrm{~h}$ \\
PIHCA & $54 \mathrm{~h}$ & $4.6 \mathrm{~h}$ \\
\hline
\end{tabular}




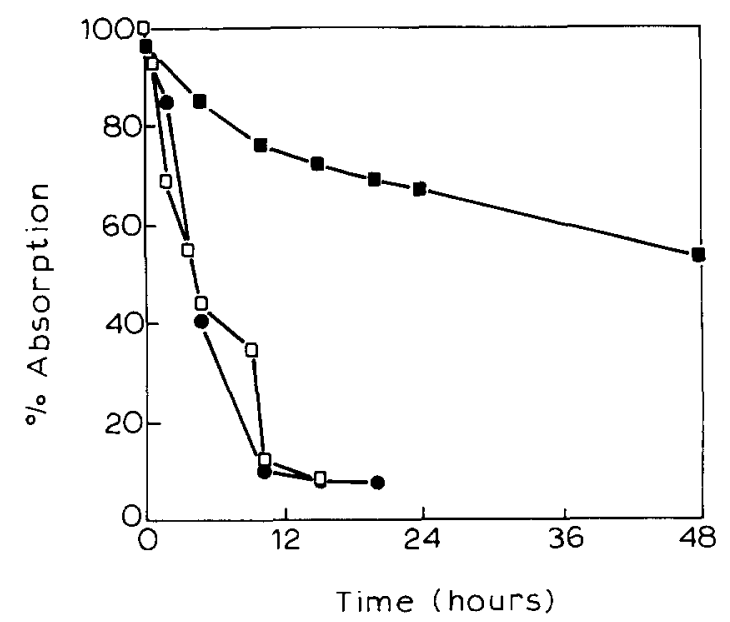

Fig. 10. Degradation of uncoated PIHCA ( $\square)$ and nanoparticles protected sterically against aggregation by coating with Poloxamer $407(\bullet)$ and Poloxamine $908(\square)$ in cell culture medium.

(Fig. 10). After thus eliminating the distortions by particle aggregation, the true degradation half-life could be determined, yielding $t_{50 \%}$ values in the range of $5 \mathrm{~h}$ (Table 4). The slowly degrading polymers PIBCA and PIHCA therefore release a low concentration of degradation products over a prolonged period of time.

\section{Conclusions}

Cyanoacrylate particles varying in alkyl chain length could be prepared of similar size $(100-300$ $\mathrm{nm}$ ) in order to minimize possible size effects during in vitro cell culture studies. For PECA, PECA-PI, PIBCA and PIHCA, identical polymerization conditions were employed to reduce differences in the surface properties of particles arising from surface-entrapped detergents or stabilizers. However, by varying the surfactant concentration in the polymerization medium, particles of different sizes could be produced for the evaluation of size effects.

The properties of the investigated particles were similar with regard to surface charge, $\zeta$ potential, interaction with charged blood components and surface hydrophobicity. The particles possessed a relatively low surface charge in the range of $2 \mu \mathrm{C} / \mathrm{cm}^{2}$ which was consistent with the $\zeta$-potential measurements. The $\zeta$-potentials in distilled water $(-6$ to $-26 \mathrm{mV})$ were observed to fall to zero at physiological salt concentrations. After intravenous injection, the particle charge in the blood is therefore supposed to be determined solely by adsorbed serum components. Since their surfaces are similar in degree of hydrophobicity, the adsorption behaviour should be identical. This assumption is in agreement with the identical adsorption of charged serum components leading to $\zeta$-potentials in serum of about $-3 \mathrm{mV}$. From these data, no differences in the in vitro interaction with cells in culture or in vivo organ distribution are a priori expected with these cyanoacrylate nanoparticles of different alkyl chain length.

The predominant mechanism of particle degradation was found to be a process of surface erosion. Degradation in cell culture medium revealed a considerable difference between the rapidly degrading PMCA and PECA and the relatively slowly degrading PIBCA and PIHCA nanoparticles. This could lead to divergence in the results obtained on further examination of the interaction with cells in culture. Particles degrading rapidly, i.e., within a few minutes, should lead to a burst release of possibly cytotoxic degradation products into the culture medium.

The incorporation of a fluorescent marker did not enhance the rate of degradation. Also, coating of particles with polymers (surface modification) affected neither the type nor the rate of degradation, as found for Poloxamine 908 and Poloxamer 407.

Further experiments are in progress to elucidate these well characterized nanoparticles in cell cultures as well as to determine the physicochemical parameters which are critical to the cellnanoparticle interaction (see the following paper (Lherm et al., 1992)).

\section{Acknowledgements}

The research was supported by the European Community within the framework of the program 
'Biotechnology'. We would like to express our sincere thanks.

\section{References}

Couvreur, P., Polyalkylcyanoacrylates as colloidal drug carrier. CRC Crit. Rev. Ther. Drug Carrier Systems, 5 (1988) 1-20.

Cummins, H.Z. and Pike, E.R., Photon correlation and Light Beating spectroscopy, NATO Advanced Study Institute Series, B3, Plenum, New York, 1973.

Cummins, H.Z. and Pike, E.R., Photon Correlation Spectroscopy and velocimetry, NATO Advanced Study Institutes Series, B23, Plenum, New York, 1976.

Delgado, G., Potkul, R.K., Treat, J.A., Lewandowski, G.S., Barter, J.F., Forst, D. and Rahman, A., A phase I/II study of intraperitoneally administered doxorubicin entrapped in liposomes in patients with ovarian cancer. $\mathrm{Am}$. J. Obstet. Gynecol., 160 (1989) 812-819.

Grislain, L., Couvreur, P., Lenaerts, V., Roland, M., DeprezDecampeneere, D. and Speiser, P., Pharmacokinetics and distribution of a biodegradable drug-carrier. Int. J. Pharm., 15 (1983) $335-345$.

Hunter, R.J., Zeta Potential in Colloid Science: Principles and Applications, Academic Press, London, 1981.

Illum, L. and Davis, S.S., Targeting of colloidal particles to the bone marrow. Life Sci., 40 (1987) 1553-1560.

Illum, L., Davis, S.S., Müller, R.H., Mak, E. and West, P., The organ distribution and circulation time of intravenously injected colloidal carriers sterically stabilized with a blockpolymer - Poloxamine 908. Life Sci., 40 (1987) 367-374.

Johnson, S.A., Thomas, N.W., Warren, M., Wilson, C.G. and Fry, J., Uptake of latex microparticles by rat hepatocytes in tissue culture. J. Pharm. Pharmacol, 38 (1986) 101p.

Kayes, J.B. and Rawlins, D.A., Adsorption characteristics of certain polyoxyethylene-polyoxypropylene block copolymers on polystyrene latex. Colloid Polym. Sci., 257 (1979) $622-629$.

Kazatchkine, M.D. and Carreno, M.P., Activation of the complement system at the interface between blood and artificial surfaces. Biomaterials, 9 (1988) 30-35.

Koppel, D.E., Analysis of macromolecular polydispersity in intensity correlation spectroscopy: The method of cumulants. J. Chem. Phys., 57 (1972) 4814-4820.

Kreuter, J., Physicochemical characterization of polyacrylic nanoparticles. Int. J. Pharm., 14 (1983) 43-58.

Lenaertz, V., Couvreur, P., Christiaens-Leyh, D., Joiris, E., Roland, M., Rollman B. and Speiser, P., Degradation of poly(isobutylcyanoacrylate) nanoparticles. Biomaterials, 5 (1984) 65-68.

Lherm, C., Etude de l'interaction des nanoparticules de polyalkylcyanoacrylates avec des fibroblastes en culture: influence des paramètres physico-chimiques sur la cytotoxicité, $\mathrm{PhD}$ thesis, Université de Paris-Sud, ChâtenayMalabry, France, 1990.

Lherm, C., Müller, R.H., Herbort, J. and Couvreur, P., Proc. 5th Int. Conf. Pharm. Technol. (APGI), 3 (1989) 81-88.

Lherm, C., Müller, R.H., Puisieux, F. and Couvreur, P., Alkylcyanoacrylate drug carriers: II. Cytotoxicity of cyanoacrylate nanoparticles with different alkyl chain length. Int. J. Pharm., 84 (1992) 13-22.

Müller, R.H., Differential opsonization - a new approach for the targeting of colloidal drug carriers. Arch. Pharm., 322 (1989) 700.

Müller, R.H., Colloidal Carriers for Controlled Drug Delivery and Targeting - Modification, Characterization and In Vivo Distribution, Wissenschaftliche Verlagsgesellschaft/ CRC Press, Stuttgart/Boca Raton, 1991.

Müller, R.H. and Heinemann, S.H., Surface modelling of microparticles as parenteral systems with high tissue affinity. In Gurny, R. and Junginger, H.E. (Eds), Bioadhesion Possibilities and Future Trends, Wissenschaftliche Verlagsgesellschaft, Stuttgart, 1989, pp. 202-213.

Müller, R.H., Lherm, C., Herbort, H. and Couvreur, P., In vitro model for the degradation of alkylcyanoacrylate nanoparticles. Biomaterials, 11 (1990) 590-595.

Müller, R.H., Lherm, C., Herbort, J. and Couvreur, P., Propidium iodide loaded polyalkylcyanoacrylate particles labelling conditions and loading capacity. Colloid Polym. Sci., 269 (1991) 147-152.

Polysciences Product Catalogue, Polysciences Inc., Warrington, U.S.A.

Van Oss, C.J., Gillman, C.F. and Neumann, A.W., Phagocytic Engulfment and Cell Adhesiveness as Cellular Surface Phenomena, Dekker, New York, 1975.

Verdun, C., Couvreur, P., Vranckx, H., Lenaerts, V. and Roland, M., Development of a nanoparticle controlled-release formulation for human use. $J$. Controlled Release, 3 (1986) 205-210.

Vezin, W.R. and Florence, A.T., In vitro heterogeneous degradation of poly( $n$-alkyl $\alpha$-cyanoacrylates). J. Biomed. Mater. Res., 14 (1980) 93-106.

Wallis, K.H. and Müller, R.H., Stabilität von Poloxamer und Poloxamine Coatingfilmen auf Polystyrol Nanopartikeln. Acta Pharm. Technol., 36 (1990) 127-132.

Wilkins, D.J. and Myers, P.A., Studies on the relationship between the electrophoretic properties of colloids and their blood clearance and organ deposition in the rat. $B r$. J. Exp. Pathol., 47 (1966) 568-576. 